\title{
Consumer Co-production in Prolonged and Complex Services: The Case of Medication Adherence in Chronically Ill Individuals
}

\author{
Jelena Spanjol, Anna S. Cui, Cheryl Nakata, Lisa Sharp, \\ Stephanie Y. Crawford, Yazhen Xiao, and Mary Beth Watson-Manheim
}

\begin{abstract}
This study examines customer co-production in a prolonged, complex, and negative service context-medication adherence in chronically ill individuals. We integrate services and medical perspectives to develop a novel theoretical framework of adherence as a nested system of co-production behaviors, characterized by temporal and scope dimensions. Utilizing a qualitative approach, our findings point to two key insights about co-production in the customer sphere. First, the enactment and form of regular-restricted, intermittent-intermediate, and irregular-expansive co-production behaviors are determined by the characteristics of the customer sphere - that is, co-production is contextualized. Second, the co-production system in the customer sphere is complex, and the different levels are interdependent. Our research contributes to the emerging literature on service co-production by elucidating the behaviors through which customers strive towards adherence. The identified co-production framework holds important implications for providers of prolonged and complex services and future research directions.
\end{abstract}

\footnotetext{
J. Spanjol $(\bowtie) \bullet$ A.S. Cui $\bullet$ C. Nakata $\bullet$ L. Sharp $\bullet$ S.Y. Crawford $\bullet$ Y. Xiao M.B. Watson-Manheim

University of Illinois at Chicago, Chicago, IL, USA

e-mail: spanjol@uic.edu; ascui@uic.edu; cnakat1@uic.edu; sharpl@uic.edu; crawford@uic.edu; yxiao21@uic.edu; mbwm@uic.edu
} 